\title{
Older Adults' Explanatory Models of Colds and Flu
}

\author{
Margo-Lea Hurwicz, Margaret Rose \\ Department of Anthropology \& Archaeology, University of Missouri Saint Louis, Saint Louis, Missouri, USA \\ Email: hurwicz@umsl.edu
}

Received 12 August 2015; accepted 21 August 2015; published 25 September 2015

Copyright (c) 2015 by authors and Scientific Research Publishing Inc.

This work is licensed under the Creative Commons Attribution International License (CC BY). http://creativecommons.org/licenses/by/4.0/

(c) $\underset{\mathrm{EY}}{\mathrm{EY}}$ Open Access

\begin{abstract}
Systematic data collection and analysis techniques were used in Los Angeles to discover older adults' shared explanatory models (EM) of the causes, prevention, symptoms, treatment and consequences of late life illnesses, including influenza and the common cold. Recorded narratives also were analyzed to illustrate similarities and differences in shared cultural knowledge of these illnesses. Consensus analyses results suggest that shared EM of influenza and the common cold are similar. Participants identified both illnesses as contagious, caused or exacerbated by bad weather, but not the result of lifestyle, aging or heredity. Other shared cultural knowledge includes that both illnesses can be treated with home remedies, over-the-counter medications and medical care; both illnesses cause discomfort but are not serious, life-threatening or disabling. Despite the similarities and the apparent merging of the two illnesses in popular thought, many older adults do distinguish them, based on symptom patterns and severity, as revealed in their transcribed narratives. Consistent with other studies, participants attribute gastrointestinal symptoms to influenza but not to colds. They do not understand the potential role of lifestyle, age and chronic conditions in etiology and onset, and they are not concerned with their vulnerability to the potential sequelae of influenza. Public health education explaining the effects of lifestyle on susceptibility and vulnerability to the flu, how to distinguish and appropriately treat colds and the flu, and when to contact physicians, is recommended for older adults. Mixed method studies can prove useful at the planning stages of such interventions.
\end{abstract}

\section{Keywords}

Older Adults, Influenza, Common Cold, Explanatory Model, Consensus Analysis

\section{Introduction}

Anthropological research has shown that the meaning given to illness by individuals and groups, and their responses in coping with it are embedded in the broader context of cultural beliefs, values and practices. Conse- 
quently, the study of peoples' cultural understandings of illness that influence their health-related actions has both theoretical importance and primary relevance for health care and the efforts of public health professionals.

Kleinman [1] [2] developed the explanatory model (EM) framework as a systematic way to focus on particular illness episodes and the explanations of cause, symptoms, treatment and prognosis given by the people experiencing them. He made a clear distinction between broadly shared cultural beliefs about health and illness in any given society and the way in which these beliefs are drawn upon and specifically adapted by individuals as they try to cope with a particular illness episode or health concern. A biomedical practitioner may have a very different, and sometimes incompatible, explanatory model (EM) for an illness compared with the patient who is seeking help. As a result, a practitioner may provide a diagnosis and prescribe a given treatment regimen, only to find that the recommendations are neither acceptable nor comprehensible within the framework of the EM being used by the patient and his or her family.

Practitioners may not even be aware that their patients have cultural understandings and individual EM that differ significantly from biomedical models. Therefore, the study of lay, cultural knowledge of specific illnesses can serve to alert biomedical practitioners that such models exist in given populations for conditions such as diabetes [3] [4], Alzheimer's disease [5], heart disease [6] and hypertension [7] [8].

Studying cultural knowledge of illnesses experienced as frequently as influenza and the common cold can provide interesting insights into routine self-care practiced by individuals and also into lay perceptions of the limitations of this self-care. Under what circumstances, for example, do people feel they should seek help for a commonly experienced illness? When and under what circumstances is a common illness perceived to be not so common? What then should be done to cope with this illness?

Such questions are relevant not only because influenza and the common cold are experienced frequently, but also because they can have serious sequelae. Serious upper respiratory infections can develop into more serious lower respiratory infections, such as bronchitis and pneumonia. And even though culturally shared knowledge may define influenza as not very serious and "just the flu", certain symptoms commonly associated with it may be in fact due to etiological processes distinct from the etiology of influenza. They may indicate the presence of a health condition that poses a very serious threat to both a patient's life and public health [9].

\section{Background}

Older adults are at greater risk than younger adults for suffering complications of influenza. Understanding how a Medicare-eligible population in the US perceives both influenza and the common cold can give useful insight into how older adults generally perceive these illnesses and how they define the limitations of self-care. This in turn may suggest avenues for better health education in areas such as the interpretation of specific symptoms and the advisability of getting flu and pneumonia vaccinations.

Helman [10] examined folk beliefs about colds, chills and fevers among patients who consulted general practitioners in a London suburb. He analyzed his own experiences as a general practitioner and interviewed other healthcare providers in the community. He found that fever and colds/chills were perceived to be very different illnesses with distinct causes, effects on the body, and required treatments.

Colds and chills were thought to be caused by exposure to dampness, cold, drafty or windy environments. Because concerted action to keep from catching colds was thought possible, by dressing warmly, eating properly and using fortifying tonics, people who caught them were blamed for behaving foolishly or irresponsibly. The symptoms of a cold, according to the folk model, included subjectively feeling cold, nasal congestion or discharge, watery eyes and "shivering, rigours, malaise [and] vague muscular aches" [10]. Consistent with beliefs about individual blame for catching a cold, the treatment was considered to be a person's own responsibility. Treatment sought "to fight cold with warmth ... by adding heat in the form of hot drinks, hot-water bottles, rest in a warm bed ... giving ... the means to generate [one's] own heat, especially by ample warm food ... as well as tonics and vitamins" [10].

While colds and chills were caused by natural forces outside of human society, fever was associated with interpersonal relationships and classified as an infectious illness caused by a "germ", "bug" or "virus" caught from other people. Catching a fever was seen to be a risk inherent in all relationships with others in society and not the fault of those who became ill. Symptoms of fever included a subjective feeling of being hot, nasal congestion and discharge, a productive cough, diarrhea and abdominal discomfort and skin rash. Treatment included expulsion of the germ, by coughing it up with the help of expectorants like tea, honey, hot water and cough 
medicine, "washing" it out by drinking lots of fluids or "sweating it out".

Helman also noted that diagnoses given by general practitioners to patients presenting with colds, chills and fever were vague and "couched in the folk idiom; for example: 'You've picked up a Germ', 'You've got a 'flu Bug', 'It's a Viral infection', and 'It's just an ordinary Cold'” [10]. He concluded that folk concepts about fever/colds/chills had not only persisted in this London suburb but also influenced the thinking and practice of healthcare providers themselves. Their diagnoses and prescribed treatments reinforced, rather than weakened, the folk illness concepts held by patients.

McCombie [11] examined the conceptions of "folk flu" among laymen and its equivalent, "viral syndrome", among healthcare providers in the southwestern United States, collecting ethnographic and epidemiological data over a three-year period while working as a communicable disease investigator for a county health department. For "most lay people", McCombie [11] points out, "flu has a much broader definition than it does for an epidemiologist, and includes illnesses with a number of clinical presentations.” Many illnesses involving nausea, vomiting and diarrhea are considered by laymen to be flu. These symptoms were seen by some as the defining characteristics of the flu, while others categorized them as more typical of a specific type of "stomach flu", in contrast to the kind of flu manifesting both respiratory and some gastrointestinal symptoms. On the other hand, laymen may perceive illnesses that have respiratory symptoms consistent with the narrower, epidemiological definition of influenza, as bad colds.

McCombie [11] also focused on the category of "viral syndrome" used by physicians and other health-care providers. This category does not correspond any more to a specific, epidemiologically defined disease than does folk flu. Physicians frequently make this diagnosis in emergency rooms and during office visits on the basis of a variety of different symptoms that include fever, headache, vomiting and diarrhea. The diagnosis of "viral syndrome" allows the practitioner to give a label to the patient's illness and, in this sense, the syndrome "is to the practitioner what flu is to the layperson" [11]. Reminiscent of Helman's discussion of the influence of the folk model on clinical thinking and practice, McCombie [11] asserts that there is "considerable semantic overlap between the diagnoses of folk flu and viral syndrome."

Baer et al. [12] found a high level of intra- and inter-cultural agreement on the causes, symptoms and treatment of the common cold, despite considerable variation in a number of demographic characteristics across five research sites. They studied four randomly chosen, representative Hispanic samples in Guatemala, Mexico, Texas and Connecticut and one randomly chosen, representative sample of middle-class Americans in Tampa, Florida. Using consensus analysis, they analyzed the responses of approximately 40 adults at each site to 142 yes/no questions regarding beliefs about colds. There was an agreement "that colds can be caused by a lack of vitamins, low resistance, exposure to drafts/wind/air, a change in the weather, not being properly clothed in cold weather, and by being around a person who has a cold" [12].

Consensus was found with regard to the more objective cold symptoms affecting the head and neck, "such as red eyes, sneezing, stuffy nose, and runny nose" [12]. Agreement decreased with regard to more subjective symptoms and symptoms that affect parts of the body other than the head and neck. Baer et al. [12] also found a high level of agreement on treatment. Informants across the five research sites agreed that one should take physician-prescribed medications and "drink orange or lemon tea, use eucalyptus or camphor balm, such as Vicks, or take vitamins" [12]. Informants agreed as well that an untreated cold could turn into bronchitis or pneumonia.

Baer et al. [13] extended this research by using the same protocol to interview physicians at the sites in Texas $(\mathrm{N}=38)$ and Mexico $(\mathrm{N}=39)$, and comparing their responses to those of the laypeople from the same location. Using consensus analysis they found that all four samples essentially shared a single EM. Nationality and level of professional training had very little effect, except for "some differences on subtheme, such as the role of hot and cold forces in the etiology of the common cold" [13].

Prior, Evans and Prout [14] found that 54 older adults from South Wales, UK who were asked to talk about the symptoms and causes of colds and flu as part of a study on flu vaccine acceptance, shared ideas about symptoms that were similar to those of the biomedical establishment. However, sematic network analysis of the interviews showed that they "integrate symptoms into a markedly different observational frame" [14]. Further, these "lay experts" emphasized the causal role of resistance and immunity at least as much as that of infection. Of particular interest is that this paper focuses on describing how "older lay people-as non-medical professionals - assign clusters of symptoms to one of the two common disease categories" [14].

The present study adds to this body of research, using both quantitative and qualitative methods to describe and compare the cultural knowledge of colds and flu shared by Medicare-eligible older adults in Los Angeles, 
during the same time period as the Baer et al. [12] [13] studies. Similarly, it used systematic data collection techniques [15] and consensus analysis [16]-[18] to identify shared explanatory models (EM) of these illnesses. In addition, participants' narratives explaining causes, preventions, treatments, and symptoms were recorded, transcribed and analyzed for common themes.

\section{Methods}

Sampling and recruitment were consistent with other research that uses consensus analysis to identify shared cultural models of illness [5] [12]. Tables providing sample size and validity estimates for different levels of agreement [17] [18] were used to determine the target sample size. Thirty participants were randomly selected for exploratory in-depth interviews about late life illnesses and their causes, preventions, treatments and symptoms from a pool of volunteers who were members of a Medicare HMO in Los Angeles. A free-listing procedure required them to recall illnesses or health conditions from memory. Free lists of causes, preventions, treatments and symptoms were elicited for each illness mentioned, and coded as present or absent. Matrices of illnesses and their attributes were analyzed to discover common themes. From their accompanying narratives, which had been recorded and transcribed, a set of sentence frames was abstracted, revised and administered to 28 of the 30 (93\%) participants using a card sorting procedure developed for this research [15] (see Table 1 for sentence frames). The objective was to identify shared cultural knowledge about each of the 30 illnesses/conditions mentioned by more than $20 \%$ of the sample. This paper focuses on their cultural knowledge, or explanatory models (EM) of the common cold (colds) and of influenza (the flu).

Table 1. Distribution of sentence completion/card sort responses and estimated answer key for colds and flu $(\mathrm{N}=28)$.

\begin{tabular}{|c|c|c|}
\hline & A Cold & The Flu \\
\hline & Consensus & Consensus \\
\hline CAUSES & $\mathrm{T}$ & $\mathrm{T}$ \\
\hline comes on or gets worse in bad Weather & $\mathrm{T}$ & $\mathrm{T}$ \\
\hline __ is Related to Drinking or Smoking & $\mathrm{F}$ & $\mathrm{F}$ \\
\hline _ is Diet-related & F & $\mathrm{F}$ \\
\hline is Age-related & $\mathrm{F}$ & $\mathrm{F}$ \\
\hline _ is related to Exercise or Weight & $\mathrm{F}$ & $\mathrm{F}$ \\
\hline is Stress-related & $\mathrm{F}$ & $\mathrm{F}$ \\
\hline is Hereditary (it runs in families) & $\mathrm{F}$ & $\mathrm{F}$ \\
\hline $\begin{array}{l}\text { TREATMENTS } \\
\text { There are effective Home Remedies for ___ _ (includes OTC medications) }\end{array}$ & $\mathrm{T}$ & $\mathrm{T}$ \\
\hline A medical Doctor can help you when you have & $\mathrm{T}$ & $\mathrm{T}$ \\
\hline $\begin{array}{l}\text { CONSEQUENCES } \\
\text { is Painful or makes you Uncomfortable }\end{array}$ & $\mathrm{T}$ & $\mathrm{T}$ \\
\hline is Serious & $\mathrm{F}$ & $\mathrm{F}$ \\
\hline _ is Life Threatening & $\mathrm{F}$ & $\mathrm{F}$ \\
\hline _ is Disabling (changes your life) & F & $\mathrm{F}$ \\
\hline First Factor Eigenvalue & 17.62 & 16.19 \\
\hline Second Factor Eigenvalue & 2.20 & 2.64 \\
\hline Eigenvalue Ratio & 8.01 & 6.14 \\
\hline Average Knowledge & 0.78 & 0.75 \\
\hline Standard Deviation & 0.14 & 0.14 \\
\hline
\end{tabular}




\subsection{Sample}

Of the 30 individuals who participated in the free-listing exercise, 26 (84\%) mentioned the common cold (colds) as an illness for which older adults are at risk, and 21 (68\%) mentioned influenza (the flu).

The socio-demographic and health characteristics of the participants are as follows. Eighteen (60\%) were female and $12(40 \%)$ were male; 9 (30\%) were between 70 and 74 years of age, $13(43 \%)$ were between 75 and 79 years, 5 (17\%) were between 80 and 84 years, and $3(10 \%)$ were 85 and older (mean age 78 years). Twelve (40\%) were married and 15 (50\%) were widowed. Twenty-six (87\%) were retired. Most of the participants were non-Hispanic white (23\% or 77\%). Four (13\%) were African-American, and $3(10 \%)$ were Hispanic American.

Twenty-three individuals (77\%) reported their health as good or excellent; 20 individuals (67\%) reported having one or more chronic conditions. Twenty-nine (97\%) had a "regular" doctor, and 30 (100\%) had seen a physician in the past year.

\subsection{Procedures ${ }^{1}$}

Interview appointments were set up to visit participants in their homes at a convenient time for them. All interviews were conducted by the first author, and lasted between one and two hours. During the first home visit, they were asked to list all the illnesses or health conditions that an adult might encounter in later life. For each illness or condition on their list, they were asked to list symptoms, causes, preventions and treatments. Thus, only a person who spontaneously mentioned the flu or colds would have been asked for more detail (see the listed items in Table 2). The responses were recorded by the first author, then coded and entered into ANTHROPAC, a computer program designed for the systematic analysis of cognitive anthropology data [16].

While listing attributes of the illnesses, participants gave narrative descriptions of the context for their judgments. These were tape-recorded and transcribed. The coded lists of attributes were independently verified from the transcriptions. Examples from the narratives were selected to illustrate the coded categories and to develop

Table 2. Distribution of free list responses for colds and flu.

\begin{tabular}{|c|c|c|}
\hline & A Cold $(\mathrm{N}=26)$ & The Flu $(\mathrm{N}=21)$ \\
\hline & Number Recalling (\%) & Number Recalling (\%) \\
\hline \multicolumn{3}{|l|}{ CAUSES } \\
\hline Germs/Viruses/Bugs & $18(69)$ & $13(50)$ \\
\hline Climate/Dress/Wet-Cold & $10(38)$ & $2(8)$ \\
\hline \multicolumn{3}{|l|}{ PREVENTIONS } \\
\hline Flu or pneumonia shot & $3(12)$ & $12(46)$ \\
\hline Home remedies (taking Vitamin C) and & $12(46)$ & $2(8)$ \\
\hline Self-care (dressing warmly) & & \\
\hline Avoid germs, people with illness & $7(27)$ & $4(15)$ \\
\hline \multicolumn{3}{|l|}{ TREATMENTS } \\
\hline Home remedies (teas, soups, toddies) & $22(85)$ & $13(50)$ \\
\hline \multicolumn{3}{|l|}{ Self-care (rest) } \\
\hline OTC medication & $13(50)$ & $8(31)$ \\
\hline Prescription medication/Physician contact & $3(12)$ & $3(12)$ \\
\hline Unspecified medication & $3(12)$ & $2(8)$ \\
\hline \multicolumn{3}{|l|}{ SYMPTOMS } \\
\hline Coughing & $17(65)$ & $5(19)$ \\
\hline Fever/Chills & $8(31)$ & $10(38)$ \\
\hline Body aching & $3(12)$ & $15(58)$ \\
\hline Runny nose & $13(50)$ & $2(8)$ \\
\hline Sneezing & $10(38)$ & $1(4)$ \\
\hline Chest congestion/Chest pain & $8(31)$ & $3(12)$ \\
\hline Headache & $6(23)$ & $5(19)$ \\
\hline Fatigue, low energy & $4(15)$ & $7(27)$ \\
\hline Gastrointestinal Symptoms & $3(12)$ & $7(27)$ \\
\hline Sore throat/Hoarse/Thickening in throat & $8(31)$ & $1(4)$ \\
\hline Watery eyes/Irritated eyes & 5 (19) & $1(4)$ \\
\hline Stuffy nose & $4(15)$ & $1(4)$ \\
\hline
\end{tabular}

${ }^{1}$ Procedures were reviewed and approved by the Institutional Review Board of the University of Missouri-St. Louis. 
sentence frames for a more systematic data collection procedure (see sentence frames in Table 1).

During a second home visit, 28 of the participants responded to the more systematic protocol. This protocol consists of 14 general sentences that apply to cultural knowledge about many illnesses, adapted from the narratives. Each sentence includes a blank into which any illness/condition name can be inserted to make it true or false. Each participant was given a set of 30 cards with the names of the most frequently mentioned illnesses or conditions printed on them. For each sentence, they were instructed to sort the cards into one pile that would make the sentence true, and another pile that would make the sentence false. For the example, "(Blank) is life threatening", cancer would most likely make the sentence true; arthritis would most likely make the sentence false. They did not have to recall from memory either the illnesses or the elements of the cultural explanatory models for the illnesses. This method efficiently produced an answer of true or false to $420(14 * 30)$ sentence/questions for each participant. These data also were entered into ANTHROPAC [16]. The participant ( $\mathrm{N}=$ 28) by question ( $\mathrm{N}=14)$ matrices for the "colds" and the "flu" cards were used in this paper.

\subsection{Operational Hypothesis}

A priori hypotheses included that bad (cold) weather would be said to cause colds, but not flu; that doctor visits would be recommended for flu but not colds; and that flu but not colds would be perceived as serious and life threatening. Hypotheses about lifestyle effects on these illnesses were also considered: Drinking and smoking, diet, exercise and stress, to the extent that they affect resistance might be perceived as risk factors. Expected results for these variables were not specified, however.

\subsection{Data Analysis}

The sentence frame/card sort data were analyzed using the Consensus Analysis procedure in ANTHROPAC [16]. This procedure analyzes a person-by-question data matrix to provide an estimate of each individual's level of cultural knowledge about a given domain. The statistical model is based on the assumption that the questions tap a shared cultural domain, and assesses whether this assumption is met. If it is met, the estimates may be used to develop a key to the culturally agreed upon answers to the questions. Two additional assumptions are that the participants answer the questions without consulting one another, and that all of the questions are equally representative of the cultural domain under consideration.

A more complete description of the analytic procedures is available elsewhere [17] [18]; however, the basic elements of the statistical analysis are presented here. A correlation matrix representing agreement among the responses of the participants is factor analyzed after a mathematical correction for guessing, and the factor loading for each informant is taken as an estimate of his/her level of cultural knowledge or expertise. For the assumption of shared cultural knowledge to be met, according to the conventions of consensus analysis, the amount of variance accounted for by the first factor must be three times larger than the amount accounted for by the second [17] [18]. If this assumption is met, a Bayesian formula is applied to these estimates (factor loadings) to determine the "culturally correct" response to each question (answer key).

The results of these analyses are presented in the following section. Next, raw tabulations of causes, preventions, treatments and symptoms coded from the narratives are summarized, and verbatim examples abstracted from the narratives are used to illustrate categories of interest. Additional narrative materials are used to further explore the differences between colds and the flu as articulated by the participants who offered such distinctions.

\section{Results}

\subsection{Consensus Analysis of Sentence Completion/Card Sort Data}

Table 1 shows the results of the sentence completion/card sort task. In both analyses the relationship between the eigen values of the first two factors is greater than 3 and the average cultural knowledge score is between 0.7 and 0.8 , with standard deviations of 0.14 . With this sample size $(\mathrm{N}=28), 99 \%$ of their answers can be said to be culturally "true" with a 0.99 confidence level. For both illnesses, each of the sentences could be reliably classified as true or false with a p-value of 0.001 .

Substantively, the most striking finding is that colds and the flu have the same general attributes. They are both contagious. They are both brought on by, and/or exacerbated by the weather, although it was hypothesized that the flu would not be. They do not result from lifestyle factors that pose health risks such as drinking, smok- 
ing, (poor) dietary practices, lack of exercise, or stress. They are not a function of aging, and they are not the result of hereditary predisposition. These older adults agree that both colds and the flu respond to home remedies, including over-the-counter medications. They also agree that a doctor can help a person with a cold or the flu; the hypothesis that medical care would not be recommended for a cold was not supported. One consequence of both of these illnesses, according to their shared cultural knowledge, is discomfort and/or pain. Neither of these illnesses is considered serious, life threatening or disabling. The hypotheses that there would be agreement that the flu was serious and life threatening were not supported, although some (29\%) participants did say that the flu might result in death.

\subsection{Categories Coded from the Free Lists}

Specific categories coded from the free list narratives are found in Table 2.

People talked about contagion in terms of germs that are transmitted and about the methods of transmission from person to person. Most of them failed to distinguish the germs that cause colds from the germs that cause the flu.

They talked about the weather or climate in terms of susceptibility, the immune system, and resistance. This type of explanation is more common for colds than for the flu. Prevention was said to involve avoiding people with germs or avoiding exposure to bad weather/climate, dressing warmly, and keeping up one's resistance. Vitamin C was thought to increase resistance to colds in particular. To prevent the flu, more than half (57\%) recommended a flu shot.

Treatment revolved around home remedies of various descriptions, but "rest, drink plenty of liquids (chicken soup, teas, orange juice) and take aspirin (or various over-the-counter cold and flu preparations)" is a good summary. Vitamin C also was specified as a treatment. Going to the doctor for colds and the flu was not often advocated spontaneously in the narratives, although in the card sort protocol, people did agree that seeking medical care would help.

Regarding the discomfort involved in these illnesses, people were much more specific in the narratives than the card sorting routine allowed them to be. Table 2 also shows the symptoms listed for each illness. The two most frequently mentioned symptoms for colds are coughing and runny nose, followed by sneezing, chest congestion, sore throat and fever/chills. The two most frequently mentioned for the flu are general body aches and fever/chills, followed by fatigue and gastrointestinal symptoms. The presence of gastrointestinal symptoms in the shared cultural knowledge about flu is interesting because the biomedical model of influenza usually does not include these symptoms [11] [14]. While there is considerable overlap of symptom categories between colds and the flu, the two illnesses are different in terms of the frequency with which symptoms are mentioned. Cold symptoms occur mainly in the upper body, while flu symptoms tend to involve the whole body.

\subsection{Illustrations from the Narratives}

In the following sections verbatim material from the free list narratives is presented to support and clarify the results reported above.

\subsubsection{Causes}

Colds and flu are contagious; you can catch them from other people. A variety of terms for the small organisms thought to cause colds and flu, including viruses, germs or "bugs", were used interchangeably.

- "Well we know [flu]'s as a result of an organism. I don’t know. Did we say a virus or did we say a microbe? I mean germ. What do we use? I guess we use a germ." (FB76)

- "You catch cold from a virus, or a bacteria or whatever." (FJ85)

Participants agreed that both colds and flu are contagious, so a good way to avoid getting ill is to stay away from people who are ill.

- "I could probably go to the grocery store and the next day I'll have a cold. I get it from somebody that's spreading germs around or something." (MIr79)

- "Stay out of crowds. That's how you can catch [colds] very easy. You don't know who's gonna sneeze right in your face.” (MJ73)

Being around children was specifically mentioned as an easy way to catch these illnesses.

- “I usually don't get a cold. The only time I get them it seems is when I'm around children that have had 
colds. And as you know little children pick this up at school and bring it home to their parents and it goes through the whole family and I usually get it too.” (FA78)

Colds and flu can be transmitted through direct physical contact between people or through touching something a sick individual has touched and left a virus, germ or "bug" on. The germ is then "picked up" by anyone who comes along afterwards and touches the same object. Some people felt that direct contact with the germ or virus was the only way to catch a cold or flu.

- "I think what's been giving us sore throats is [we] drink [from] a glass and somebody had a sore throat and the germs was left on there and we drink out of it. Or eat on a fork or spoon. We give one another colds and sore throats that way. So now, I tell my wife to scald all dishes and spoons and we scald them with hot water to get rid of the germs. We just pass it around that way until we scald them out. Colds, sore throat, all come from hygiene on dishes and spoons and glasses.” (MB75)

- "If people with a cold touch something and you touch that. Like they used to say, when you wash your hands, always take the toilet paper and wipe off the soap first because the person in front of you might have left a cold virus on it. Don't touch anything that somebody who has a cold, touches. And wash your hands and your face frequently." (MA81)

Others felt that germs or viruses could also be spread through the air.

- "Because you can get [a cold] if somebody sneezes and it comes over and you breathe it. So I'd say it's an airborne virus, probably. Well, you stay out of crowded rooms where people [have colds]. Don't breathe the air that somebody that has a cold does." (MA81)

Colds and flu come on or get worse in bad weather. Weather, climate and dress were mentioned most often in conjunction with colds. Although not many described weather conditions as direct causes of illness, most agreed that illness episodes could be prolonged or worsened by these factors. Some emphasized exposure to general weather conditions, such as cold, dampness, wind and rain.

- "When I lived in Boston, I had colds. But the climate was very damp there. I think the dampness causes colds.” (FJ85)

- "I had gone in the swimming pool with the kids and then I, they wanted to go to the park, and then I took them down to the park. And, it was such a windy place. I tried to get out of the wind and that's where I know I caught the cold. But that happened about 4:00 in the afternoon. Then by 6:00 I started feeling bad.” (FA73)

- "I've lived in England, in Europe, in Canada, and here. People like to have a window open, fresh air. And when that air is full of water, snow, and cold you breathe in that air, you have a tendency of catching a cold very easy. If it's raining, instead of getting in, taking cover, you have a tendency to stand there in the rain. And it gets through you and your body gets wet, your clothing gets wet, and you cannot be warm if you're soaking wet. If your feet are wet, you're going to catch a cold fast.” (MJ73)

Several also mentioned exposure to changes in temperature, winds and drafts without adequate clothing as causal or risk factors.

- "Like I was saying, I'd go out at night without my hat. Boy, I can get [a cold] fast that way. Sudden change in temperature will get me. At my age, I think my blood is thin. And I have to put on more clothes than I used to. Wear the hat and the jacket. Keep my extra blanket on the bed at night. Bundle up. But if I get out and forget to take a jacket after it gets [to be] nighttime? [That's] All it takes.” (MB75)

- "You're working hard, you're perspiring and windy, you don't have any protection on you. At least that's what they tell me-cause of the cold. Well, dress warm in wintertime [to prevent colds]. If you're perspiring, stay out of the draft. I haven't had a cold in a long time. A couple days ago I started getting a cough. The weather changing and all that crap. And then I go outside without anything on, you know.” (MIt76)

- "You can get [colds] when you sweat and getting in a draft, I guess. That's probably one of the instances. Don't wear enough clothes, I guess.” (MA93)

Constitution, resistance, and vulnerability play a role. Some mentioned the strength of a person's constitution as a factor that interacts with exposure to contagious viruses and/or weather conditions. A person can, for example, be exposed to germs or adverse weather conditions and still not become ill if their constitution is strong enough. Some felt that people could be naturally resistant to illness.

- "I have a cold maybe once every five years. I'm not susceptible to that sort of thing. For which I'm very grateful. Even if everybody around me has fever, sneezing, everything, I don't.” (FJ75)

- 'I don't catch colds like that because I'm strong. I'm a strong person. I don't mean I'm so strong necessarily that I couldn't get sick or anything like that. I'm strong in mind and I think the mind has a lot to do with that.” 
(FJ85)

On the other hand, they could be particularly vulnerable because their resistance is low.

- "You know older people used to say if you're not dressed warm or something like that you can get a cold or something but I don't think so. You know the body just can't take it. I think it's because, well, it's a breakdown in whether the body is able to withstand some of the germs that's out there.” (FB82)

\subsubsection{Prevention}

The recommendations given for preventing colds and flu were consistent with the major causes given for them. Contagion as a cause was reflected in recommendations to stay away from people already sick with a cold or flu and to wash one's hands more often, as well as any objects an ill person may have touched. Injunctions to dress appropriately, stay out of the rain and avoid drafts are consistent with beliefs about the role of weather conditions and temperature changes in the course of these illnesses.

Flu shots prevent flu. Consistent with the belief that viruses and germs are the primary cause, many people suggested getting shots to prevent the flu, sometimes noting that vaccines have been developed against specific strains of flu virus. Several individuals had hypotheses about the relationship of their flu shots to their subsequently contracted colds.

- "But I get my flu shot every year-both of us. We get our flu shot. One year we got our flu shots and my wife came down with a cold and she said, 'I think they gave me a cold'.” (MIr79)

- "We've both had shots this winter. We didn’t have flu, but we did have a cold. But the shot didn’t cause it.” (FGe85)

- "I get the flu shot now. When I started taking them I realized that it did help me a lot. So I had my flu shot, but about Christmas time, I got a cold. Now, when I get a cold after having the flu shot, I don't feel bad. I have a cold and I have mucous and all of that and I cough occasionally, but I don't feel bad.” (FA76)

Vitamin C prevents colds. In the absence of vaccines to prevent colds, methods of building up and maintaining bodily resistance to illness were recommended. Taking vitamins, particularly vitamin C, was mentioned frequently as a constitution-strengthening cold prevention. Indeed, taking vitamin $\mathrm{C}$ during cold season seemed to function as an equivalent to getting shots for the flu.

- "But I take a large amount of vitamin C and I take vitamin C all year, but small amounts, and then I increase it long about time for when I get a cold. And when I get a cold, believe it or not, it's a couple of weeks in and around Christmas and so I kind of speed up my vitamin C about a month before. Hoping, yes, hoping that if I get a cold I won't be real sick, you know.” (FB76)

- “[To prevent colds] I do take an excess amount of vitamin C as a rule. Ordinarily I don't take the vitamin C. I just take one vitamin. [When do you start taking it?] When I know that they have the colds then I start. It seems to prevent it with me, the vitamin C.” (FA78)

\subsubsection{Treatment}

There are effective home remedies for colds and the flu. They include taking aspirin, cough syrup and other over-the-counter medications from the drugstore. Also mentioned were drinking lots of fluids such as herbal tea, soda, fruit juices, hot lemonade and lemon and honey in water and drinking alcohol such as brandy, bourbon or whiskey. Eating light foods, going to bed and resting and using humidifiers and inhalants to clear one's sinuses as well as sweating out a cold or flu were mentioned as well.

Only a few of the many home remedies were seen as specific to either colds or the flu. In the case of flu accompanied by stomach problems some advised not eating at all. Taking vitamin $\mathrm{C}$ was mentioned only for colds, consistent with the frequent mention of vitamin $\mathrm{C}$ as a means of preventing colds. Chicken soup is another popular home remedy that is thought to be particularly effective for colds.

- "I think chicken soup helps colds and maybe even pneumonia. You know it sounds silly when you talk chicken soup cure. It doesn't cure; what it does is help your body to heal. When a person eats chicken soup, with the chicken in it and the vegetables as well, any person would get well from that.” (FJ85)

- "I believe with all my heart and soul and so do my children that there's no substitute for chicken soup for a cold." (FJ75)

Some described very basic home treatments involving rest, drinking fluids and aspirin. Others described elaborate, well-orchestrated regimens.

- The first sign of sore throat or, if I've been around people who have colds and I think I might be coming 
down with one, I make my pot of chicken soup right away. One jigger, or a little more, of apricot brandy, taken just before you go to bed, turn the electric blanket on high and just sweat it out during the night and wake up in the morning and you'll have no symptoms of a cold anymore." (FB77)

A medical doctor can help alleviate the symptoms of both colds and the flu. However, these illnesses will run their natural course if not treated. People only recommended seeking, or sought the assistance of a doctor when they thought they were very, very sick. This was especially true in the case of colds, and a number of them stated flatly that they or people in general, did not go to doctors for a cold.

- "Well, with a cold I wouldn’t see a doctor period. You don't go at the first sign of a cold because you think, "This is ridiculous. I can take care of it myself." (FJ77)

- "I'd say that most people when they get a cold, they don't spend the money and go to the doctor, they go in the drugstore and say, 'Hey, I've got a cold. Give me something'.” (MA81)

Going to the doctor involved considering how long a cold or flu had persisted in the face of self-treatment, how bad they felt and how serious they thought the illness might be.

- "Well, three days in bed and lots of juice and aspirin and a cold, what do you call it? Various cold remedies. I, there again, it's a matter of degree. If you can catch [the cold] quick, you can knock it out yourself. If you can't, then you have to go to the doctor.” (MA82)

- "Well, last time [I had the flu] I went to the doctor. He gave me some medicine and I took that and it was all gone, I quit taking it. The rest of the flu went away by itself. I had to break the spirit of the flu, I guess. That's the worst one I ever had in my life, I think. [What made you decide to go to the doctor?] Well, it got so bad I could hardly stand it anymore." (MA93)

\subsubsection{Symptoms/Course}

The symptoms of colds and the flu cause discomfort. Although there is considerable overlap among the specific symptoms listed in Table 2, the frequencies do conform to the often observed pattern that colds are thought to occur mostly in the upper part of the body, while the flu is a whole body illness. This method of coding does not, however, reveal the perceived relationship between the two illnesses. For example, one individual described a head or chest cold as a symptom of the flu.

- "Well, there's, let's see, what is this type of flu that's going around? It's more or less feeling tired I guess and they have a chest cold. It's in their chest and they're, gee my son had it. He had a head cold with it." (FA81)

Others had trouble differentiating between a cold and the flu, sometimes seeing them as different stages of the same illness.

- "Probably the beginning of the cold then starts going into a flu. [So one turns into the other?] Yeah, they're related, I think." (MIt76)

- "In other words, a cold develops into flu. You get sore muscles. You get sore throat, running eyes, running nose, cough. And once a cold develops into a flu, the flu then develops into a fever and this is where you have got to start taking care of it, because once a fever gets very strong, it's a very dangerous thing." (MM74)

- "Colds, I guess that would be the same as the flu, I guess. I lump them together there because I can't tell much difference, to be honest with you." (MM74)

Some did see the flu and colds as having different underlying organisms. And one understood that there are many strains of influenza.

- " "I think the flu bug is more vicious, myself, than a regular cold bug." (MM74/2)

- "Well apparently there are different strains of influenza. And I know that last year the flu shot they gave was for an Asiatic flu and this year it's for a different strain. But when does it stop being a cold and become flu? I would guess it would be only when you were exposed to that particular virus that caused it. I wouldn't think a cold would turn into the flu. I think that a lot of people think it does. But if what I read is true, that the flu now is caused by specific strains. That wouldn't say that a common cold could turn into the flu." (MA81)

\subsubsection{Distinguishing Criteria}

Many people thought that colds and the flu could be distinguished according to specific criteria. These included the duration and the severity of the illness.

- "If you're still weak and miserable after a week, you have the flu. A cold you get over within a week. But if it's the flu, you're still weak and miserable even after the symptoms have departed-the primary symptoms." 
(MJ74)

- "I think with the flu, don't you have more aches and pains?” (FA73)

They also distinguished between colds and flu according to specific symptoms. While some thought that a fever could be a symptom of a cold, others drew the line between colds and flu precisely on the basis of whether or not there was a temperature.

- "If you don't have a temperature with your cold, you did not have the flu. But if you had the temperature, you had the flu.” (FGe85)

- "With the flu you run a temperature. Generally you run a temperature." (FJ77)

Most individuals who mentioned body aches saw them as characteristic of the flu (see Table 2), with aches that are associated with a cold being restricted to the head, throat and chest only.

- "But a cold could be just on the chest where your chest is, you know, congested. Look like something is pressing on your chest and it hurts if you cough or something like that. And of course a head cold means that it's all in your head." (FB82)

Finally, gastrointestinal symptoms were cited by many as distinguishing features of the flu.

- "Well, with the flu you have running of the bowels and you can't eat and you feel like throwing up." (FIt76)

- "Well, let's see, the flu is, like when you have diarrhea and stomach flu and all that. But when I get the cold, I don't have the stomach flu or diarrhea or anything like that. Just comes out my nose. The flu is when you have the cold, the stomach system and diarrhea and vomiting. That's the flu; that's the flu.” (MIr79)

- "Well, flu most people get bones are aching and um, nausea. Very seldom are people upset with their stomach with the cold. Headaches and fever, what have you, but this flu ... Like this flu, some people were nauseous, other people said their joints just ached something terrible and I think that, two basic differences between just a basic cold and the flu." (FIr74)

\section{Discussion}

In combination with the findings of Helman [10], McCombie [11], Baer et al. [12] [13] and Prior [14], the results of this study of older adults in Los Angeles confirm the existence and persistence of widely shared cultural knowledge about both the flu and colds. The shared explanatory models identified by this body of research include both ideas that are, and ideas that are not, part of the biomedical/epidemiological explanatory models (EM) for these illnesses.

The most striking finding in the systematic data presented here is that colds and the flu have the same profile when examined at the level of general illness attributes. The consensus analysis shows that they are identical: both are contagious and brought on by or exacerbated by the weather. Neither is the effect of poor lifestyle choices, aging or heredity. They can be treated with home remedies and over-the-counter medications, but they also respond to medical care. Both cause discomfort, but they are not serious, life-threatening or disabling.

These results do not support the hypotheses that flu would not be thought to come on or get worse in bad weather; that a doctor visit would not be expected to help a cold; or that the flu would be considered serious and life-threatening.

The narratives provide more detailed information on what people think about contagion and weather. Colds and flu result from organisms that are transmitted by personal contact and/or through the air. Children are often the vectors. Exposure to bad weather lowers resistance, or makes things worse once the illness episode has started. Significantly, the participants in this study mentioned the importance of specific weather-related factors such as dampness, wind and bodily exposure to cold and to sudden temperature changes, echoing humoral (hot/cold) explanations found in other studies [10] [12]-[14].

In contrast to the clear distinction that Helman's [10] study participants made between colds and illnesses characterized by fever, the data presented in this paper suggest a merging of these two categories in popular thought. Helman [10] hypothesized that younger people were already beginning to merge these categories due to the influence of germ theory. The Los Angeles data most likely reflect the extent to which ideas about germs have been integrated into both the "cold/chills" and "flu/fever" categories in the English-speaking world. Current public health messages in the American mass media about the approach of "cold and flu season" and popular advertisements touting the effectiveness of "cold and flu medications" no doubt reflect and reinforce the cultural understanding that both colds and flu are caused by organisms transmitted through human contact.

Yet weather, temperature and dress continue as prominent factors in these older adults' causal/preventive 
perceptions of colds and, to a lesser extent, the flu. Individuals who maintain some of the more traditional beliefs may attempt to reconcile them with newer ideas about organisms by modifying them. Thus, weather and the failure to dress properly are seen as lowering resistance and increasing susceptibility to germs rather than causing colds and flu directly. Or, they are seen as making symptoms more serious or making an illness episode last longer. Thus, the research reported here supports observations made by other researchers that Americans have folk beliefs about illnesses that diverge from biomedical models, just as commonly as do other populations more characteristically described as holding such beliefs [11]-[13].

However, in considering the potential contribution of this work to health education and policy, it is equally important to note the influence of biomedical knowledge on the shared cultural knowledge elicited from older adults in Los Angeles. While differences from the content of public health messages about these illnesses may be used as an indicator of what needs to be targeted in future educational interventions, similarities may be used as an indicator of areas where public health information already has been received.

The narratives provided information about distinctions between colds and flu in terms of symptoms, their frequency, intensity and duration. When the results presented above are compared to descriptions of colds and flu presented in books available in the popular press, there are both similarities and differences. For example, Griffith, in The Complete Guide to Symptoms, Illnesses and Surgery for People over 50 [19] first lists upper respiratory symptoms, such as a runny nose, sore throat and coughing as indicators of a cold. These are among the most frequently mentioned symptoms for colds on study participants' free lists. General body symptoms such as chills/fever and body aches, the most frequently free-listed for the flu, are also listed first by Griffith. On the other hand, a relevant discrepancy from Griffith is the persistent idea that gastrointestinal symptoms are part of the flu. However, as Prior [14] points out, the study participants may not be wrong.

Further, the causes and exacerbating factors endorsed in Los Angeles only partially reflect the biomedical knowledge presented by Griffith [19]. Although they agreed that person-to-person contact and exposure to bad weather could cause or exacerbate colds, and that the flu could be spread by personal contact, the study participants did not agree that lifestyle factors, such as stress, overwork, and poor nutrition could be risk factors for both illnesses. Further, they did not agree with Griffith that smoking could be a risk factor for colds.

Two risk factors for flu mentioned by Griffith, a recent acute illness and/or ongoing chronic illness also are missing from the narratives. The study participants also did not appear to understand the potential role of aging in susceptibility to both colds and flu. Therefore, public health efforts should be made to educate older adults that they are more vulnerable to infectious diseases than younger adults, and that they are also more likely to suffer serious complications associated with them. Specifically, they should be taught that lifestyle can play a role in the course of acute upper respiratory infections and that gastrointestinal symptoms may indicate an illness other than the flu.

Regarding medical interventions, Griffith [19] recommends preventive injections for the flu, as did many of the participants in their free list narratives. He further agrees with the older adults from Los Angeles that a flu episode warrants a visit to the doctor. He suggests that colds that cannot be distinguished from the flu should be brought to medical attention, but he does not recommend medical care for colds. A study [20] that investigated physician consensus on the "appropriateness" of seeking medical care for a variety of symptoms/illnesses also suggests that while physicians recommend medical care for the flu, they do not recommend that older adults seek medical care for colds. Therefore, it would useful to design health education materials to tell people how to distinguish between colds and the flu, and how to act appropriately at home to alleviate cold symptoms while the body is recovering.

With regard to the methodology and focus of the research described above, it is clear that this kind of investigation is useful in describing older adults' explanatory models of colds and the flu, including how they might differ from those of biomedical practitioners. Consensus analyses of systematically collected data and thematic analyses of open-ended narratives each made a unique contribution. Health educators and policy makers should therefore find the periodic review of mixed method studies of this kind useful during the planning phases of their work.

\section{References}

[1] Kleinman, A. (1978) Concepts and a Model for the Comparison of Medical Systems as Cultural Systems. Social Science and Medicine, 12B, 85-93. http://dx.doi.org/10.1016/0160-7987(78)90014-5

[2] Kleinman, A. (1980) Patients and Healers in the Context of Culture. University of California Press, Berkeley.

[3] Garro, L. (1995) Individual or Societal Responsibility? Explanations of Diabetes in an Anishinaabe (Ojibway) Com- 
munity. Social Science and Medicine, 40, 37-46. http://dx.doi.org/10.1016/0277-9536(94)00125-D

[4] Garro, L. (1996) Intracultural Variation in Causal Accounts of Diabetes: A Comparison of Three Canadian Anishinaabe (Ojibway) Communities. Culture, Medicine and Psychiatry, 20, 381-420. http://dx.doi.org/10.1007/BF00117086

[5] Schrauf, R.W. and Iris, M. (2011) Using Consensus Analysis to Investigate Cultural Models of Alzheimer's Disease. In: Kronenfeld, D.B., Bennardo, G., de Munck, V.C. and Fischer, M.D., Eds., A Companion to Cognitive Anthropology, Blackwell Publishing Limited, Oxford, 548-568. http://dx.doi.org/10.1007/BF00117086

[6] Good, B. (1977) The Heart of What's the Matter: The Semantics of Illness in Iran. Culture, Medicine and Psychiatry, 1, 25-58. http://dx.doi.org/10.1007/BF00114809

[7] Blumhagen, D. (1982) The Meaning of Hyper-Tension. In: Chrisman, N.J. and Maretzki, T.W., Eds., Clinically Applied Anthropology, D. Reidel, Dordrecht, 297-323. http://dx.doi.org/10.1007/978-94-010-9180-0 11

[8] Garro, L. (1988) Explaining High Blood Pressure: Variation in Knowledge About Illness. American Ethnologist, 90, 98-119. http://dx.doi.org/10.1525/ae.1988.15.1.02a00070

[9] Heurtin-Roberts, S. (1993) 'High-Pertension'-The Uses of a Chronic Folk Illness for Personal Adaptation. Social Science and Medicine, 37, 285-294. http://dx.doi.org/10.1016/0277-9536(93)90260-B

[10] Helman, C. (1978) "Feed a Cold, Starve a Fever"-Folk Models of Infection in an English Suburban Community and Their Relation to Medical Treatment. Culture, Medicine and Psychiatry, 2, 107-138. http://dx.doi.org/10.1016/0277-9536(93)90260-B

[11] McCombie, S.C. (1987) Folk Flu and Viral Syndrome: An Epidemiological Perspective. Social Science and Medicine, 25, 987-993. http://dx.doi.org/10.1016/0277-9536(87)90003-7

[12] Baer, R., Weller, S., Pachter, L., Trotter, R., de Alba Garcia, J., Glazer, M., et al. (1999) Cross-Cultural Perspectives on the Common Cold: Data from Five Populations. Human Organization, 58, 251-260. http://dx.doi.org/10.17730/humo.58.3.n4413t15t4220567

[13] Baer, R., Weller, S., De Alba Garcia, J.G. and Salcedo Rocha, A.L. (2008) Cross-Cultural Perspectives on Physician and Lay Models of the Common Cold. Medical Anthropology Quarterly, 22, 148-166. http://dx.doi.org/10.1111/j.1548-1387.2008.00012.x

[14] Prior, L., Evans, M.R. and Prout, H. (2011) Talking about Colds and Flu: The Lay Diagnosis of Two Common Illnesses among Older British People. Social Science \& Medicine, 73, 922-928. http://dx.doi.org/10.1016/j.socscimed.2010.09.054

[15] Hurwicz, M. (2008) From Free List to Consensus: Explanatory Models of Late Life Illness. Paper Presented to the American Anthropological Association, San Francisco. (Unpublished)

[16] Borgatti, S. (1992) ANTHROPAC 4.0. Analytic Technologies, Columbia.

[17] Romney, A.K., Weller, S. and Batchelder, W.H. (1986) Culture as Consensus: A Theory of Culture and Informant Accuracy. American Anthropologist, 88, 313-338. http://dx.doi.org/10.1525/aa.1986.88.2.02a00020

[18] Weller, S.C. (2007) Cultural Consensus Theory: Applications and Frequently Asked Questions. Field Methods, 19, 339-368. http://dx.doi.org/10.1177/1525822X07303502

[19] Griffith, H. (1992) Complete Guide to Symptoms, Illness \& Surgery for People Over 50. Berkley Publishing Group, New York.

[20] Hurwicz, M. (1995) Physician's Norms and Health Care Decisions of Elderly Medicare Recipients. Medical Anthropology Quarterly, 9, 211-235. http://dx.doi.org/10.1525/maq.1995.9.2.02a00060 